\title{
Adaptable and Adaptive Information Access for All Users, Including the Disabled and the Elderly
}

\author{
Josef Fink, Alfred Kobsa, and Andreas Nill \\ GMD FIT, German National Research Center for Information Technology, \\ St Augustin, Germany
}

\begin{abstract}
The tremendously increasing popularity of the World Wide Web indicates that hypermedia is going to be the leading online information medium for the years to come and will most likely be the standard gateway to the "information highway". Visitors of web sites are generally heterogeneous and have different needs, and this trend is likely even to increase in the future. The aim of the AVANTI project is to cater hypermedia information to these different needs by adapting the content and the presentation of web pages to each individual user. The special needs of elderly and handicapped users are also considered to some extent. Our experience from this research is that adaptation and user modeling techniques that have so far almost exclusively focused on adapting interactive software systems to "normal" users also prove useful for adaptation to users with special needs.
\end{abstract}

\section{User Needs in a Metropolitan Information System}

The aim of the AVANTI project (see the AVANTI Home Page) is to develop and evaluate a distributed system which provides hypermedia information about a metropolitan area (e.g. about places of interest, transportation, and public services) for a variety of users, including tourists, residents, travel agency clerks, elderly people, blind persons, wheelchair-bound people, and users with slight forms of dystrophy. The system is to be used at people's homes, public information kiosks and in travel agencies, each with different hardware platforms, software environments, network speeds, and environmental surroundings.

From interviews that we conducted and from literature (e.g. Zeiner et al., 1995) we determined that our users partially differ in their aims, interests, experience and abilities. Here are a few examples concerning elderly and handicapped users:

- In general, elderly users prefer cultural and historic information, while young travelers are more interested in shopping and entertainment possibilities, as well as sports events.

- For vision-impaired users, the screen display should be enlarged. For blind users, the modality of the presented information must be changed to tactile and/or audio output. Moreover, additional orientation and navigation aids (e.g., tables of contents, indices) are helpful for this user group (Kennel et al., 1995).

- For wheelchair-bound users, information concerning the accessibility of premises (e.g., the existence, location, and the dimensions of ramps and elevators, the type and width of doors) is important and should therefore be automatically provided. 
- For users with slight forms of dystrophy, including many elderly persons, the graphical interface (i.e., the interaction objects and associated manipulation techniques) should be made less sensitive to erratic hand movements.

\section{Adaptable and Adaptive System Behavior}

In order to cater to different user needs, information systems can be tailored manually by the user or system administrator, or automatically by the system based on assumptions about the user. Both features, adaptability and adaptivity (Oppermann, 1995), will be provided by the AVANTI system:

- Within the user interface, special I/O devices (e.g., macro mouse, Braille display, speech synthesizer), visual and non-visual interface objects, and associated interaction techniques are integrated (cf. Savidis et al., 1995);

- Within hypermedia pages, the information content, information modality, information prominence, orientation and navigation aids, search facilities, and links to other hypermedia pages are adapted (cf. Brusilovsky, 1996).

Whereas the first group of adaptations aims at enabling and improving the overall access to information systems, the second group of adaptations aims at individualizing one specific hypermedia system. In the remainder of this paper we will focus on adaptivity within hypermedia pages, emphasizing adaptation to elderly and certain types of handicapped users.

\section{User and Usage Model}

In order to provide user-oriented adaptivity, a user model is maintained by the AVANTI system which contains assumptions about relevant user characteristics, including:

- motoric and sensory abilities, such as the user's ability to overcome physical obstacles (e.g. stairs, narrow passageways), to perceive visually presented information, and to select objects on the user interface;

- interests and preferences, such as the user's interest in accessibility information for premises (e.g., the availability of ramps and elevators), detailed information about the history of points of interests, or preferences for certain presentation modalities (e.g., graphics, video);

- domain knowledge with respect to the contents of the information system;

- competence in handling computers and the AVANTI system.

Different methods for acquiring assumptions about the user have been discussed in the literature (Chin, 1993). In AVANTI, assumptions are based on the following sources of information:

- An initial interview allows for the acquisition of primary assumptions about the user and is therefore a valuable source of information for initially assigning the user to certain user subgroups (see the 'stereotypes' below).

- Certain dialog actions performed by the user can be exploited for the acquisition of primary assumptions. For instance, if the user requests an explanation for a technical term then it can be assumed that he or she is not familiar with it (Kobsa et al., 1994). 
- Based on primary assumptions about the user and additional information about the application domain, the system can draw inferences in order to acquire further assumptions about the user. For instance, if the user more than once requests detailed information on the history of some church, he or she can be assumed to be interested in churches, and similar detailed information will henceforth automatically be provided.

- Stereotypes contain assumptions about interesting characteristics of user subgroups (e.g., tourists, blind users). They can be applied to a user if certain preconditions are met.

A subcomponent of the user model, the usage model, additionally contains a record of the user's interaction with the system as well as information about the location and environmental surroundings of the user's terminal, its technical abilities, and the network quality.

\section{Discussion}

Computer access for the handicapped has been a research issue for many years. Considerable efforts have been put into making software systems usable by people for whom they were not originally designed (e.g. access to graphical user interfaces for visually or manually impaired users), and into developing databases with dedicated information for the handicapped (e.g., information on wheelchair accessibility of public transportation, or verbal descriptions of paintings in museums) to supplement existing data collections that lack this essential information for people with special needs. These solutions are mostly restricted to fairly small classes of handicapped users and are therefore usually quite expensive due to the small number of potential customers. It seems, however, that techniques from the area of useradapted interaction can be extended in such a way that they permit to some extent the tailoring of generic interactive software systems to all users, including many handicapped and elderly people. This approach not only is theoretically more satisfactory but may also be economically more viable than isolated dedicated solutions.

\section{References}

AVANTI Home Page. Available at http://www.gmd.de/fit/hci/projects/avanti.

Brusilovsky, P. (1996). Methods and techniques of adaptive hypermedia. User Modeling and UserAdapted Interaction 6:87-129.

Chin, D. N. (1993). Acquiring user models. Artificial Intelligence Review 7:185-197.

Kennel, A., Perrochon, L., and Darvishi, A. (1996). WAB: World-Wide Web access for blind and visually impaired computer users. New technologies in the education of the visually handicapped. ACM SIGCAPH Bulletin 55: 10-15.

Kobsa, A., Müller, D., and Nill, A. (1994). KN-AHS: An adaptive hypertext client of the user modeling system BGP-MS. Proceedings of the Fourth International Conference on User Modeling, 99-105.

Oppermann, R. (1995). Adaptive user support: Ergonomic design of manually and automatically adaptable software. Hillsdale, $\mathrm{NJ}$ : Erlbaum.

Savidis, A., and Stephanidis, C. (1995). Developing dual user interfaces for integrating blind and sighted users: The Homer UIMS. Proceedings of the CHI'95 Conference on Human Factors in Computing Systems, 106-113.

Zeiner, M., Harrer, B., and Bengsch, L. (1995). Städtetourismus in Deutschland. Deutscher Fremdenverkehrsverband, Bonn. 